\title{
Fecal Calprotectin is an Accurate Tool and Correlated to Seo Index in Prediction of Relapse in Iranian Patients With Ulcerative Colitis
}

\author{
Seyed Vahid Hosseini ${ }^{1}$; Peyman Jafari ${ }^{2}$; Seyed Alireza Taghavi ${ }^{3}$; Ali Reza Safarpour ${ }^{3,{ }^{*}}$; Abbas \\ Rezaianzadeh ${ }^{4}$; Maryam Moini ${ }^{3}$; Manoosh Mehrabi ${ }^{3}$ \\ ${ }^{1}$ Colorectal Research Center, Department of Surgery, Shiraz University of Medical Sciences, Shiraz, IR Iran \\ ${ }_{2}^{2}$ Biostatistic Department, Shiraz University of Medical Sciences, Shiraz, IR Iran \\ ${ }^{3}$ Gastroenterohepatology Research Center, Department of Internal Medicine, Shiraz University of Medical Sciences, Shiraz, IR Iran \\ ${ }^{4}$ Laparoscopy Research Center, Department of Surgery, Shiraz University of Medical Sciences, Shiraz, IR Iran \\ *Corresponding Author: Ali Reza Safarpour, Gastroenterohepatology Research Center, Department of Internal Medicine, Shiraz University of Medical Sciences, Shiraz, IR Iran. Tel: \\ +98-7112357282, Fax:+98-7112307594, E-mail: asafarpour@sums.ac.ir
}

Received: August 16, 2014; Revised: September 25, 2014; Accepted: November 9, 2014

\begin{abstract}
Background:The natural clinical course of Ulcerative Colitis (UC) is characterized byepisodes of relapse and remission. Fecal Calprotectin (FC) is a relatively new marker of intestinal inflammation and is an available, non-expensive tool for predicting relapse of quiescent UC. The Seo colitis activity index is a clinical index for assessment of the severity of UC.

Objectives: The present study aimed to evaluate the accuracy of FC and the Seo colitis activity index and their correlation in prediction of UC exacerbation.

Patients and Methods: In this prospective cohort study, 157 patients with clinical and endoscopic diagnosis of UC selected randomly from 1273 registered patients in Fars province's IBD registry center in Shiraz, Iran, were followed from October 2012 to October 2013 for 12 months or shorter, if they had a relapse. Two patients left the study before completion and one patient had relapse because of discontinuation of drugs. The participants' clinical and serum factors were evaluated every three months. Furthermore, stool samples were collected at the beginning of study and every three months and FC concentration (commercially available enzyme linked immunoassay) and the Seo Index were assessed. Then univariate analysis, multiple variable logistic regression, Receiver Operating Characteristics (ROC) curve analysis, and Pearson's correlation test (r) were used for statistical analysis of data.

Results: According to the results, 74 patients (48.1\%) relapsed during the follow-up (33 men and 41 women). Mean \pm SD of FC was $862.82 \pm$ $655.97 \mu \mathrm{g} / \mathrm{g}$ and $163.19 \pm 215.85 \mu \mathrm{g} / \mathrm{g}$ in relapsing and non-relapsing patients, respectively $(\mathrm{P}<0.001)$. Multiple logistic regression analysis revealed that age, number of previous relapses, FC and the Seo index were significant predictors of relapse. ROC curve analysis of FC level and Seo activity index for prediction of relapse demonstrated area under the curve of $0.882(\mathrm{P}<0.001)$ and $0.921(\mathrm{P}<0.001)$, respectively. Besides, FC level of $341 \mu \mathrm{g} / \mathrm{g}$ was identified as the cut-off point with $11.2 \%$ and 79.7\% relapse rate below and above this point, respectively. Additionally, Pearson correlation coefficient $(r)$ between FC and the Seo index was significant in prediction of relapse $(r=0.63, \mathrm{P}<0.001)$. Conclusions: As a simple and noninvasive marker, FC is highly accurate and significantly correlated to the Seo activity index in prediction of relapse in the course of quiescent UC in Iranian patients.
\end{abstract}

Keywords:Calprotectin; Ulcerative Colitis; Relapse

\section{Background}

Inflammatory Bowel Diseases (IBD), including Ulcerative Colitis (UC) and Crohn's Disease (CD) are chronic intestinal disorders of unknown etiology with a typically relapsing and remitting course (1). Most patients with chronic UC run a relapsing course. The reasons of such relapses remain unknown. Diarrheal episodes, abdominal pain, occasional rectal bleeding, anorexia, and anemia with or without fever are complains during relapse periods $(2,3)$. Besides, many of patients need hospitalization and intensive cares in the course of exacerbation. For evaluation and risk stratification of patients for exacerbations, using a simple, noninvasive and inexpensive test would be highly desirable. An ideal marker would be sensitive, thereby reliably detecting intestinal inflammation, yet affording a good specificity that avoids unnecessary investigations (4). Fecal Calprotectin (FC) an important granulocyte cytosolic protein, is closely correlated to fecal excretion of 111-indiumlabeled leucocytes (5). Several studies investigated the value of FC in prediction of relapse in non-symptomatic patients (6-10). Gisbert et al. included 89 CD and 74 UC patients in clinical remission, and found that sixteen patients (9.8\%) experienced a clinical relapse within 12 months of follow-up, and FC level at enrollment was higher in patients with clinical relapse (11). In addition, Tibble et al. reported significantly higher FC levels in relapsing compared to non-relapsing patients with UC (7). Furthermore, there are some valuable indices for assess- 
Hosseini SV et al.

ment of activity of the disease such as the Seo index (12), which uses multiple biomarkers and clinical symptoms to identify the severity of UC.

\section{Objectives}

The present study aimed to evaluate the accuracy of FC and the Seo colitis activity index and their correlation in prediction of UC exacerbation.

\section{Patients and Methods}

This prospective cohort study was conducted from 1 October 2012 to 1 October 2013, when we followed 157 UC outpatients diagnosed according to the Truelove criteria (13). Two patients left the study before completion and one patient had relapse because of discontinuation of drugs. The number of patients in this study was the same sample size originally used in the previous large study to assess time to relapse in patients with UC. All patients had been in clinical remission for at least three months and were selected through systematic sampling from 1273 registered patients, which the sampling interval was approximately eight (1273/157). All 1273 patients (sampling frame) had been registered in governmental IBD registry, related to Shiraz University of Medical Sciences, Shiraz, Iran (14). The registry center was established in 1989 and has registered and followed IBD patients actively in Fars province, in southern Iran. Gastroenterohepatology research center has a complete access to patients and their paper and electronic files and this study was conducted in the above research center. Clinical remission in this study was defined as the Seo index lower than 120 or lack of rectal bleeding and having normal stool frequency $(15,16)$. Patients who had used nonsteroidal anti-inflammatory drugs (NSAIDs), in oral, topical or enema routs, within three months prior to the study or were pregnant were excluded from the study, since these conditions are known to be associated with elevated FC levels. This study was approved by the ethics committee of Shiraz University of Medical Sciences in 21 January 2012 (code: 6024).

\subsection{Follow-up Plan}

After complete explanation of reasons, objectives and method of our study to patients, all of them signed an informed consent form before enrolment. The patients were followed for 12 months from October 2012 to October 2013 , or shorter if they had relapse, with serum, stool and clinical evaluation performed every three months. All patients with definite relapse after accurate recording of relapse date were excluded from the study. Clinical relapse was defined as elevated Seo activity index higher than 220 (17) or worsening of symptoms (including abdominal pain, diarrhea with or without blood and rectal bleeding) sufficient to require a change in therapy (increasing the dose, changing the current drug (s), addition of steroids, hospitalization or surgery) (18).

\subsection{Variable Measurements}

Demographic and disease related variables were obtained from patients according to registry questionnaire by one trained general practitioner. In first and each follow-up visit, UC severity questionnaire, according to the Seo index and drugs consumption questionnaire were completed.

\subsection{Laboratory Investigations}

Before sampling, all enrolled patients were called and the importance of study and their samples was recalled for them properly. Then, blood and stool samples were taken from each patient at the beginning of study and every three months. CBC analysis including WBC and platelet counts was performed according to standard laboratory procedure using automated full diff analyzer. Hemoglobin ( $\mathrm{Hb}$ ) and erythrocyte sedimentation rate (ESR) were evaluated by routine laboratory methods. These four variables are measured routinely in pathobiologic laboratory and their results have well validity. For these variable and ones described below, all measuring apparatus were calibrated and checked with other control and standard samples to ensure that there were no personal or equipment errors. Serum iron concentration was determined by chemical colorimetric method (Pars Azmoon, Tehran, Iran) with a sensitivity of $5 \mu \mathrm{g} / \mathrm{g}$. This is a simple spectrophotometrical method, which measures the Fe content of each specimen by measuring the absorbance of sample in 600 nm using standard curve (19). Serum albumin (Alb) levels were determined by kinetic colorimetric method (Biosystems, SA, Spain) with a sensitivity of $1.1 \mathrm{~g} / \mathrm{L}$ (20). FC was determined using a commercially available enzyme-linked immunosorbent assay (Buhlmann Laboratories AG, Schonenbuch, Switzerland), which measures quantitative calprotectin (21). The measuring range of test was 10-600 $\mu \mathrm{g}$ calprotectin/g feces with intra- and inter-assay coefficients of $4.7 \%$ and $4.1 \%$, respectively. The calprotectin cut-off level representing a positive value was equal or greater than $50 \mu \mathrm{g} / \mathrm{g}$ as stated by the manufacturer.

\subsection{Statistical Analysis}

Quantitative variables were shown as mean \pm SD and qualitative ones were expressed as frequency (\%). Univariate comparisons were performed using independent student t-test, Mann-Whitney and chi square tests. Additionally, Pearson correlation test (r) was applied to determine the correlations between FC and the Seo index. The primary outcome of study was relapse in the course of UC. First, all independent variables including age, gender, cigarette smoking, appendectomy, duration of disease before the study, extra-intestinal manifestations, months of remission before the study, number of previous relapses, the Seo index, Hb, ESR, WBC, Alb, platelets, and FC were analyzed in univariate 
analyses to determine the potential association with the outcome variable. Then all associations with $\mathrm{P}<0.1$ were included in multiple logistic regression with forward stepwise method. Multicolinearity was also evaluated by calculation of variance inflation factor. Afterwards, the ROC curve analysis was performed to evaluate the accuracy of FC and the Seo index in prediction of relapse in patients with ulcerative colitis. All analyses were performed using SPSS statistical software version 16 (Inc. Chicago, IL). P value $\leq 0.05$ was considered as statistically significant.

\section{Results}

Our study included 157 patients with UC at the beginning, but three participants withdrew during the study. Mean follow-up time of patients was 232 days. Our patients followed for one year were $48.7 \%$ female (mean age: $42.48 \pm 11.22$ years, range: $20-69$ years) and $51.3 \%$ male (mean age: $41.81 \pm 10.82$, range: $21-83$ years) and no significant differences were observed with respect to their gender distribution $(\mathrm{P}=0.70)$. According to the results, 64 non-relapsing (80\%) and 62 relapsing (83.8\%) participants received Mesalazine $(\mathrm{P}=0.65)$. Univariate comparisons of quantitative variables in relapser and non-relapser UC participants are shown in Table 1. Accordingly, the relapsers had significantly lower age, duration of disease and duration of remission before the study, $\mathrm{Hb}$ and $\mathrm{Alb}$, but higher number of previous relapses, the Seo index, ESR, WBC and FC levels compared to non-relapsers ( $\mathrm{P}<0.05)$. The univariate comparison of categorical variables between relapsers and non-relapsers UC patients is presented in Table 2 . The results demonstrated a significant difference between relapser and non-relapser patients concerning female gender and cigarette smoking $(\mathrm{P}=0.1, \mathrm{OR}=1.68, \mathrm{P}=0.01$, $\mathrm{OR}=2.55$, respectively). The results of multiple logistic regression disclosed that FC, age, number of previous relapses and the Seo index were the best variables for predicting relapse in UC patients (Table 3). The ROC curve analysis for the Seo index (the area under the curve, AUC, $0.921 \pm 0.023 ; \mathrm{P}<0.001$ ) and FC (AUC, 0.882 $\pm 0.030 ; \mathrm{P}<0.001)$ are shown in Figure 1. ROC curve and AUC were drawn and calculated, respectively, by a nonparametric method using SPSS software. Based on ROC curve analysis, FC levels of equal to or greater than 341 $\mu \mathrm{g} / \mathrm{g}$ feces had remarkable sensitivity and specificity in prediction of relapse (sensitivity $=80 \%$, specificity $=89 \%$, positive predictive value $=86.76 \%$, negative predictive value $=82.56 \%$, positive likelihood ratio $=7.09$, negative likelihood ratio $=0.23$ ). The sensitivity and specificity for prediction of relapse were high in patients with the Seo index of equal to or greater than 148.3 (82\% and 91\%, respectively and positive predictive value $=89.71 \%$, negative predictive value $=84.886 \%$, positive likelihood ratio $=9.42$, negative likelihood ratio $=0.19$ ). The maximum values of the Youden's index for calprotectin and the Seo index revealed appropriate results ( 0.74 and 0.71 , respectively). Relapse rates below and above the cut off points are presented in Table 4 . The Pearson correlation coefficient $(r)$ revealed a significant relationship between FC values and the Seo index for prediction of relapse (Table 5) $(\mathrm{r}=0.637, \mathrm{P}<0.001)$.

Table 1. Comparison of Quantitative Clinical and Demographic Characteristics in Univariate Analysis of Relapser and non-Relapser UC Patients ${ }^{a}$

\begin{tabular}{|c|c|c|c|}
\hline & Relapsers $(n=74)$ & Non-Relapsers $(\mathbf{n}=\mathbf{8 0})$ & P Value \\
\hline \multicolumn{4}{|l|}{ Quantitative variables } \\
\hline Age, $y$ & $36.90 \pm 8.89$ & $46.97 \pm 10.56$ & $<0.001$ \\
\hline Duration of disease before study, mo & $88.89 \pm 57.91$ & $109.88 \pm 64.82$ & 0.036 \\
\hline Duration of remission before study, mo & $37.39 \pm 32.67$ & $55.02 \pm 45.36$ & 0.007 \\
\hline Number of previous relapses & $5.02 \pm 3.29$ & $2.22 \pm 1.90$ & $<0.001$ \\
\hline Seo index & $278.07 \pm 142.32$ & $118.07 \pm 33.46$ & $<0.001$ \\
\hline $\mathbf{H b}, \mathrm{g} / \mathrm{L}$ & $13.36 \pm 1.77$ & $14.25 \pm 2.08$ & 0.005 \\
\hline ESR, $\mathbf{m m} / \mathbf{h}$ & $21.09 \pm 13.30$ & $13.23 \pm 8.49$ & $<0.001$ \\
\hline Alb, $\mathbf{g} / \mathbf{L}$ & $4.16 \pm 0.48$ & $4.64 \pm 0.25$ & $<0.001$ \\
\hline WBC, $\mathbf{1 0}^{9} / \mathrm{L}$ & $7.28 \pm 1.78$ & $5.38 \pm 1.10$ & $<0.001$ \\
\hline Platelets, $\mathbf{1 0}^{9} / \mathrm{L}$ & $268.43 \pm 20.2$ & $228.58 \pm 49.7$ & 0.09 \\
\hline FC, $\mu \mathrm{g} / \mathrm{g}$ & $862.82 \pm 655.97$ & $163.19 \pm 215.85$ & $<0.001$ \\
\hline Iron, $\mu \mathrm{g} / \mathrm{g}$ & $13.06 \pm 1.36$ & $13.11 \pm 1.32$ & 0.794 \\
\hline
\end{tabular}

a Data are presented as mean \pm SD. 
Hosseini SV et al.

\begin{tabular}{|c|c|c|c|c|c|}
\hline Categorical Variables & Relapsers $(n=74)$ & Non-Relapsers $(\mathbf{n}=\mathbf{8 0})$ & P Value & OR & 95\% CI \\
\hline Gender & & & & & $0.88-3.14$ \\
\hline Male & $33(41.8)$ & $46(58.2)$ & 0.10 & 1.68 & \\
\hline Female & $41(54.7)$ & $34(45.3)$ & & & \\
\hline Cigarette smoking & & & & & $1.16-5.61$ \\
\hline Non smokers & $23(31.1)$ & $12(15)$ & 0.01 & 2.55 & \\
\hline Smokers & $51(68.9)$ & $68(85)$ & & & \\
\hline Appendectomy & & & & & $0.024-2.02$ \\
\hline Yes & $4(5.4)$ & $1(1.2)$ & 0.14 & 0.22 & \\
\hline No & $70(94.6)$ & $79(98.8)$ & & & \\
\hline Extra intestinal manifestations & & & & & $0.668-2.41$ \\
\hline Yes & $29(39.2)$ & $36(45)$ & 0.46 & 1.27 & \\
\hline No & $45(60.80)$ & $44(55)$ & & & \\
\hline Positive family history & & & & & $0.43-3.14$ \\
\hline Yes & $8(10.8)$ & $10(12.5)$ & 0.74 & 1.17 & \\
\hline No & $66(89.2)$ & $70(87.5)$ & & & \\
\hline
\end{tabular}

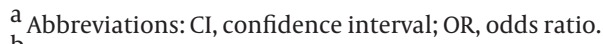

${ }^{\mathrm{b}}$ Data are presented as No. (\%).

Table 3. Cut-Off and Relapse Rates of Fecal Calprotectin (FC) and the Seo Index ( $\mathrm{n}=154)^{\mathrm{a}, \mathrm{b}}$

\begin{tabular}{lccc}
\hline Variables & Cut-Off & Relapse Rate Below Cut-Off & Relapse Rate Above Cut-Off \\
\hline FC, $\mu$ g/g & 341 & 11.2 & 79.7 \\
Seo index & 148.3 & 17.6 & 82.4 \\
\hline
\end{tabular}

a Abbreviations; FC, Fecal Calprotectin; CI, Confidence Interval; OR, Odds Ratio.

$\mathrm{b}$ Data are Presented as \%.

Table 4. The Variables Which Remained in the Final Multiple Logistic Regression Model to Predict Relapse in Patients With Ulcerative Colitis

\begin{tabular}{lcccc}
\hline Parameters & P Value & OR & \multicolumn{2}{c}{$\mathbf{9 5 \% \text { CI }}$} \\
\cline { 4 - 5 } & & & Lower & Upper \\
\hline FC & 0.001 & 8.13 & 2.33 & 28.35 \\
\hline Age & 0.002 & 9.24 & 2.29 & 37.24 \\
$\begin{array}{l}\text { Number of previous } \\
\text { relapses }\end{array}$ & 0.024 & 4.22 & 1.21 & 14.75 \\
Seo index & $<0.001$ & 52.77 & 11.86 & 234.76 \\
\hline
\end{tabular}

Table 5. The Area Under the Curve of FC and Seo Index

\begin{tabular}{lcc}
\hline Variables & Area Under Curve & PValue \\
\hline Fecal calprotectin & 0.882 & $<0.001$ \\
Seo index & 0.921 & $<0.001$ \\
\hline
\end{tabular}


Hosseini SV et al.

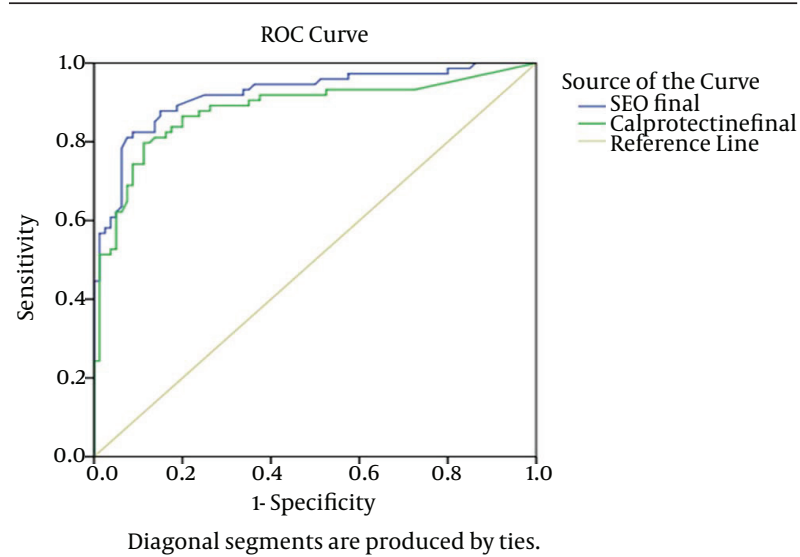

Figure 1. The ROC Curve Analysis on the Abilities of Fecal Calprotectin (FC) and the Seo Index in Prediction of Relapse in Ulcerative Colitis (UC) Patients

\section{Discussion}

In this prospective population based cohort study with a large number of patients, we evaluated the predictive ability of FC as a marker of relapse compared to the Seo index in an Iranian population of UC patients during four periods of follow-up. Our 12-month follow-up study demonstrated that in patients with UC, FC concentration was a strong predictor of clinical relapse. Moreover, the cutoff level of $341 \mu \mathrm{g} / \mathrm{g}$ had the highest combined sensitivity and specificity as a predictor of relapse in UC. Besides, the results revealed a significant correlation between FC and the Seo index in prediction of relapse. Several studies previously investigated the value of FC measurement in IBD (22-25). In addition, some studies demonstrated the predictive ability of FC measurement as a biomarker for relapse in UC $(6,8,26-28)$. However the number of study patients was relatively small and less than 100 , in all the abovementioned reports. On the other hand, one of the advantages of our study was the large number of enrolled UC patients, which enhances the power of results. As UC and $C D$ have different inflammatory patterns, the value of FC in predicting relapse may be different for the two diseases. Based on the studies conducted on the value of FC in predicting IBD relapse, FC seemed to be a relatively sensitive and specific marker of relapse in UC(29). Recently, Kallel et al. found an $18 \%$ clinical relapse while following $53 \mathrm{CD}$ patients for 12 months. They also observed that the mean calprotectin levels were significantly higher in the relapse group compared to non-relapse group (381 $\mu \mathrm{g} / \mathrm{g}$ vs. $155 \mu \mathrm{g} / \mathrm{g}$, respectively). In addition, they showed that a cut-off level of $340 \mu \mathrm{g} / \mathrm{g}$ for FC provided a sensitivity of $80 \%$ and a specificity of $91 \%$ in prediction of relapse, which was correlated to an 18 -fold increased risk (30). In a prospective multicenter study, Gisbert et al. assessed 163 IBD patients ( 89 CD, 74 UC) in clinical remission and found that the risk of relapse was $30 \%$ if calprotectin level was over $150 \mu \mathrm{g} / \mathrm{g}$ and $7 \%$ if the level was below $150 \mu \mathrm{g} / \mathrm{g}$
(11). In another study conducted by Costa et al., the risk of relapse within 12 months was increased by 14 -folds in UC patients with FC levels $>150 \mu \mathrm{g} / \mathrm{g}$ (5). It has been reported that some patients in remission may have low intestinal inflammation. Besides their FC values might be higher, but does not usually exceed $150 \mu \mathrm{g} / \mathrm{g}$ (11). Furthermore, Nakov et al. reported no significant differences between patients in remission and those in the control group concerning the average FC levels, and that the levels above $250 \mu \mathrm{g} / \mathrm{g}$ were correlated to disease relapse in the next month (31). The calculated cut-off level in the current study $(341 \mu \mathrm{g} / \mathrm{g})$ was relatively higher compared to what reported by Gisbert et al. $(150 \mu \mathrm{g} / \mathrm{g})(11)$, Costa et al. $(150 \mu \mathrm{g} / \mathrm{g})(5)$ and Nakov et al. (250 $\mu \mathrm{g} / \mathrm{g})(31)$. However, was similar to that mentioned by Kallel et al. $(340 \mu \mathrm{g} / \mathrm{g})$ (30) with good specificity and sensitivity in prediction of relapse in UC patients. In one meta-analysis conducted by Mao et al. on six prospective studies, pooled sensitivity of 0.77 , pooled specificity of 0.71 and pooled AUC of 0.78 were reported for predictive capacity of FC in UC relapse (29). Finally, Nikolaou reported that clinical indices of disease activity in IBD did not adequately reflect the degree of inflammation in remission or prior to diagnosis. However, elevated calprotectin level in these patients was more efficient than the Seo index in relapse prediction (32). Large sample size, prospective population base of the study, enough time for presentation of relapse (the major outcome of the study), measuring major quantified and qualified variables in one study and evaluation of the effect of each variable on the outcome of study and introducing new cut-off point for calprotectin and the Seo index for prediction of relapse were the major strong points of our study. Because of financial limitations, we could not check some important variables such as interleukins and histopathological grade of the intestinal mucosa. Our study results were perfectly in line with all the above mentioned previously published data. We concluded that FC was currently the best available single surrogate marker for predicting relapse in UC patients. According to the present study results, FC had a good linear correlation with Seo UC activity index during patients' follow-up. These results are of practical importance as calprotectin is remarkably stable in stools for up to seven days at room temperature, enabling sample collection at home even in patients who live in long distance from referral centers. Finally, our results encouraged application of FC measurement in clinical practice (FC levels of equal or greater than $341 \mu \mathrm{g} / \mathrm{g}$ feces) to evaluate the risk of clinical relapse in patients with UC in Iranian patients.

\section{Acknowledgements}

This study was extracted from the dissertation of Dr. Ali Reza Safarpour supported by Shiraz University of Medical Sciences (Grant number: 6495). Hereby, the authors would like to thank Ms. Mansoorabadi for assistance in data collection. We are also grateful for Ms. A. Keivan- 
shekouh at the Research Improvement Center of Shiraz University of Medical Sciences for improving the use of English in the manuscript.

\section{Authors' Contributions}

Hosseini SV: Primary idea, final review and approval of the final draft. Taghavi A.: Primary idea, final review and approval of the final draft and data collection. Jafari P.: Primary idea, final review and approval of the final draft and statistical analysis. Moeeini: Primary idea, final review and approval of the final draft and Data collection. Rezaeianzadeh A.: Design, final review and approval of the final draft and statistical analysis. Mehrabi: writing, data collection, final review and approval of the final draft. Safarpour A R: Primary idea, final review and approval of the final draft, statistical analysis, writing and data collection.

\section{Financial Disclosure}

The authors reported receiving research grants and consulting fees from Shiraz University of Medical Sciences.

\section{Funding/Support}

\section{Shiraz University of Medical Sciences.}

\section{References}

1. Yamamoto T, Shiraki M, Bamba T, Umegae S, Matsumoto K. Fecal calprotectin and lactoferrin as predictors of relapse in patients with quiescent ulcerative colitis during maintenance therapy. Int J Colorectal Dis. 2014;29(4):485-91.

2. Riley SA, Mani V, Goodman MJ, Lucas S. Why do patients with ulcerative colitis relapse? Gut.1990;31(2):179-83.

3. Stange EF, Travis SP, Vermeire S, Beglinger C, Kupcinkas L, Geboes $\mathrm{K}$, et al. European evidence based consensus on the diagnosis and management of Crohn's disease: definitions and diagnosis. Gut. 2006;55 Suppl 1:i1-15.

4. Burri E, Beglinger C. Faecal calprotectin -- a useful tool in the management of inflammatory bowel disease. Swiss Med Wkly. 2012;142:w13557.

5. Costa F, Mumolo MG, Ceccarelli L, Bellini M, Romano MR, Sterpi $\mathrm{C}$, et al. Calprotectin is a stronger predictive marker of relapse in ulcerative colitis than in Crohn's disease. Gut. 2005;54(3):364-8.

6. Canani RB, de Horatio LT, Terrin G, Romano MT, Miele E, Staiano $\mathrm{A}$, et al. Combined use of noninvasive tests is useful in the initial diagnostic approach to a child with suspected inflammatory bowel disease. J Pediatr Gastroenterol Nutr. 2006;42(1):9-15.

7. Tibble JA, Sigthorsson G, Foster R, Forgacs I, Bjarnason I. Use of surrogate markers of inflammation and Rome criteria to distinguish organic from nonorganic intestinal disease. Gastroenterology. 2002;123(2):450-60.

8. Fagerberg UL, Loof L, Myrdal U, Hansson LO, Finkel Y. Colorecta inflammation is well predicted by fecal calprotectin in children with gastrointestinal symptoms. J Pediatr Gastroenterol Nutr. 2005;40(4):450-5

9. Jeffery J, Lewis SJ, Ayling RM. Fecal dimeric M2-pyruvate kinase (tumor M2-PK) in the differential diagnosis of functional and organic bowel disorders. Inflamm Bowel Dis. 2009;15(11):1630-4.

10. Manz M, Burri E, Rothen C, Tchanguizi N, Niederberger C, Rossi $\mathrm{L}$, et al. Value of fecal calprotectin in the evaluation of patients with abdominal discomfort: an observational study. BMC Gastroenterol. 2012;12:5.

11. Gisbert JP, Bermejo F, Perez-Calle JL, Taxonera C, Vera I, McNicholl AG, et al. Fecal calprotectin and lactoferrin for the prediction of inflammatory bowel disease relapse. Inflamm Bowel Dis. 2009;15(8):1190-8.
12. Seo M, Okada M, Yao T, Ueki M, Arima S, Okumura M. An index of disease activity in patients with ulcerative colitis. Am J Gastroenterol.1992;87(8):971-6.

13. Truelove SC, Witts LJ. Cortisone in ulcerative colitis. Br Med J 1955;2(4947):1041.

14. Taghavi SA, Safarpour AR, Hosseini SV, Noroozi H, Safarpour M Rahimikazerooni S. Epidemiology of Inflammatory Bowel Diseases (IBD) in Iran: A review of 740 patients in Fars province, Southern Iran. Ann Colorectal Res. 2013;1(1):17-22.

15. Jarnerot G, Hertervig E, Friis-Liby I, Blomquist L, Karlen P, Granno $\mathrm{C}$, et al. Infliximab as rescue therapy in severe to moderately severe ulcerative colitis: a randomized, placebo-controlled study. Gastroenterology. 2005;128(7):1805-11.

16. Higgins PD, Schwartz M, Mapili J, Krokos I, Leung J, Zimmermann EM. Patient defined dichotomous end points for remission and clinical improvement in ulcerative colitis. Gut. 2005;54(6):782-8.

17. Turner D, Seow CH, Greenberg GR, Griffiths AM, Silverberg MS Steinhart AH. A systematic prospective comparison of noninvasive disease activity indices in ulcerative colitis. Clin Gastroenterol Hepatol. 2009;7(10):1081-8.

18. Henriksen M, Jahnsen J, Lygren I, Sauar J, Kjellevold O, Schulz T, et al. Ulcerative colitis and clinical course: results of a 5-year population-based follow-up study (the IBSEN study). Inflamm Bowel Dis. 2006;12(7):543-50.

19. Thomas L. Clinical Laboratory Diagnostics. 1th edFrankfurt: THBook Verlagsgesellschaft; 1998.

20. Otsuji S, Shibata H, Umeda M. Turbidimetric immunoassay of serum C-reactive protein. Clin Chem.1982;28(10):2121-4.

21. Jahnsen J, Røseth AG, Aadland E. [Measurement of calprotectin in faeces]. Tidsskrift for den Norske laegeforening: tidsskrift for prak tisk medicin, ny raekke. 2009;129(8):743-5.

22. Carroccio A, Iacono G, Cottone M, Di Prima L, Cartabellotta F, Cavataio F, et al. Diagnostic accuracy of fecal calprotectin assay in distinguishing organic causes of chronic diarrhea from irritable bowel syndrome: a prospective study in adults and children. Clin Chem. 2003;49(6 Pt 1):861-7.

23. Wassell J, Dolwani S, Metzner M, Losty H, Hawthorne A. Faecal calprotectin: a new marker for Crohn's disease? Ann Clin Biochem. 2004;41(Pt 3):230-2.

24. Schroder O, Naumann M, Shastri Y, Povse N, Stein J. Prospective evaluation of faecal neutrophil-derived proteins in identifying intestinal inflammation: combination of parameters does not improve diagnostic accuracy of calprotectin. Aliment Pharmaco Ther. 2007;26(7):1035-42.

25. Schoepfer AM, Trummler M, Seeholzer P, Criblez DH, Seibold F. Accuracy of four fecal assays in the diagnosis of colitis. Dis Colon Rectum. 2007;50(10):1697-706.

26. Bunn SK, Bisset WM, Main MJ, Golden BE. Fecal calprotectin as a measure of disease activity in childhood inflammatory bowel disease. J Pediatr Gastroenterol Nutr. 2001;32(2):171-7.

27. Kolho KL, Raivio T, Lindahl H, Savilahti E. Fecal calprotectin remains high during glucocorticoid therapy in children with inflammatory bowel disease. Scand $J$ Gastroenterol. 2006;41(6):720-5.

28. Diamanti A, Panetta F, Basso MS, Forgione A, Colistro F, Bracc $\mathrm{F}$, et al. Diagnostic work-up of inflammatory bowel disease in children: the role of calprotectin assay. Inflamm Bowel Dis 2010;16(11):1926-30.

29. Mao R, Xiao YL, Gao X, Chen BL, He Y, Yang L, et al. Fecal calprotectin in predicting relapse of inflammatory bowel diseases: a meta-analysis of prospective studies. Inflamm Bowel Dis 2012;18(10):1894-9.

30. Kallel L, Ayadi I, Matri S, Fekih M, Mahmoud NB, Feki M, et al. Fecal calprotectin is a predictive marker of relapse in Crohn's disease involving the colon: a prospective study. Eur J Gastroenterol Hepatol. 2010;22(3):340-5.

31. Nakov VN, Penchev PI, Shishenkov MT, Nakov RV, Kundurzhiev TG. Fecal Calprotectin for Assessment of Inflammatory Activity, Monitoring of Therapy and Prediction of Relapse in Ulcerative Colitis. J Gastroenterol Hepatol Res. 2012;1(11):297-302.

32. Nikolaou AM. Calprotectin: A sensitive marker of intestinal inflammation. Annals of Gastroenterology. Ann Gastroenterol. 2004 